\title{
1 Alteration and stability of complex macromolecular amino acid precursors in 2 hydrothermal environments
}

3

4

Walaa Elmasry ${ }^{1}$, Yoko Kebukawa ${ }^{1 *}$, Takeo Kaneko ${ }^{1}$, Yumiko Obayashi ${ }^{1 \dagger}$, Hitoshi Fukuda ${ }^{2}$, Yoshiyuki Oguri ${ }^{3}$, and Kensei Kobayashi ${ }^{1}$

*Corresponding author: kebukawa@ynu.ac.jp

${ }^{1}$ Department of Chemistry and Life Science, Graduate School of Engineering Science, Yokohama National University, 79-5 Tokiwadai, Hodogaya-ku, Yokohama 240-8501, Japan

${ }^{2}$ Technical Department, Tokyo Institute of Technology, 2-12-1 Ookayama, Meguro-ku Tokyo, 152-8550, Japan

${ }^{3}$ Laboratory for Advanced Nuclear Energy, Institute of Innovative Research, Tokyo Institute of Technology, 2-121 Ookayama, Meguro-ku, Tokyo, 152-8550, Japan

${ }^{\dagger}$ Current affiliation: Center for Marine Environmental Studies, Ehime University, 3 Bunkyo-cho, Matsuyama, Ehime, 790-8577, Japan

Date of submission: January 31, 2020

\section{Abstract}

The early Solar System comprised a broad area of abiotically created organic compounds, including interstellar organics which were integrated into planetesimals and parent bodies of meteorites, and eventually delivered to the early Earth. In this study, we simulated interstellar complex organic compounds synthesized by proton irradiation of a gas mixture of $\mathrm{CO}, \mathrm{NH}_{3}$, and $\mathrm{H}_{2} \mathrm{O}$, which are known to release amino acids after acid hydrolysis on the basis of Kobayashi et al. (1999) who reported that at the first stage of chemical evolution, the main compounds formed abiotically are complex organic compounds with high molecular weights. We examined their possible hydrothermal alteration and stabilities as amino acid precursors under high temperature and pressure conditions simulating parent bodies of meteorites by using an autoclave. We reported that all samples treated at $200-300{ }^{\circ} \mathrm{C}$ predominantly released glycine and alanine, followed by $\alpha$-aminobutyric acid, and serine. After heating, amino acid concentrations decreased in general; however, the recovery ratios of $\gamma$-aminobutyric acid increased with temperature. The interstellar complex organic analog could maintain as amino acid precursors after being treated at high temperature $\left(200-300^{\circ} \mathrm{C}\right)$ and pressure $(8-14 \mathrm{MPa})$. However, the molecular structures were altered during heating to form organic compounds that are more stable and can survive in elevated hydrothermal conditions.

Keywords Meteorite, Hydrothermal alteration, Amino acid precursor, Autoclave. 


\section{Sources of organic matters in early Earth}

The study of the origin of life, besides the evolution of the Earth's environments, helps us realize why the Earth became suitable to live in and why terrestrial life has continued for billions of years. Endogenous synthesis and exogenous delivery of organic compounds are suggested to be two sources of the chemical evolution of the early solar system, and organic compounds delivered by either source are considered necessary for the origin of life.

\section{Endogenous supply of organic compounds}

Many experiments have been conducted, including the historic spark discharge experiment, the Miller-Urey experiment, to reconstruct the origin of life on Earth. Miller proved that under highly reduced conditions, various organic compounds, including amino acids, could be obtained by spark discharges (Miller 1953; Miller and Urey 1959). Other investigators showed that bioorganic compounds could be synthesized from strongly reducing starting mixtures using heat (Harada and Fox 1964), UV radiation (Sagan and Khare 1971; Bar-Nun and Hartman 1978), shock waves (Bar-Nun et al. 1970), and laser-induced plasma energy (Scattergood 1989). Moreover, volcanic outgassing and hydrothermal vents provide alternative sites for organic synthesis (Yanagawa and Kobayashi 1989). On the other hand, it was suggested that they were minor contributors for organic synthesis, for example, submarine hydrothermal vents do not seem to presently synthesize organic compounds more complex than simple hydrocarbons such as $\mathrm{CH}_{4}$ and ethane (McCollom et al. 2010). It was also claimed that they decompose them over short time spans ranging from seconds to a few hours (Cleaves 2012). Prebiotic formation of bioorganics in hydrothermal vents is still controversial.

However, the predominant view in the last several years has been that the primitive Earth atmosphere was only "weakly reducing", composed of a mixture of carbon dioxide, nitrogen, and water, coupled with small quantities of carbon monoxide and hydrogen (Walker 1977; Kasting and Brown 1998). Given this, the Miller-Urey-type synthesis in the primitive Earth atmosphere would have been only a minor contributor to the formation of prebiotic organic compounds (Stribling and Miller 1987). Kobayashi and coworkers showed that amino acid precursors could be synthesized from weakly reducing gas mixtures (e.g., carbon dioxide, carbon monoxide, nitrogen, and water) by irradiation with high-energy protons (Kobayashi et al. 1998; Kobayashi et al. 1999). Thus, endogenous production of organic compounds in a weakly reducing atmosphere could be possible by the action of cosmic rays, but their production rate might have been limited.

\section{Extraterrestrial delivery of organic compounds}

Extraterrestrial infall through comets, meteorites, and interplanetary dust particles supplied considerable amounts of organic carbon to the surface of the Earth (Oró 1961; Anders 1989; Chyba and Sagan 1992; Pizzarello and Shock 2010). Carbonaceous chondrites, a primitive subgroup of stony meteorites, are mainly composed of silicates but contain abundant carbon (up to $4 \%$ ) which is mostly present as organic materials. The Murchison 
meteorite is the most well-studied carbonaceous chondrite for organic matter which is classified as insoluble macromolecular materials $(\sim 70 \%)$ and simpler soluble compounds $(\sim 30 \%)$ such as amino acids and carboxylic acids (e.g., Pizzarello et al. 2006). Murchison extracts contain over one hundred amino acids including $\alpha-, \beta-, \gamma-$, $\delta$-, and $\varepsilon$-amino acids, and eight of these amino acids are also found in proteins, namely glycine (Gly), alanine (Ala), proline, valine, leucine, isoleucine, aspartic acid (Asp), and glutamic acid, in addition to numerous other compounds encountered in terrestrial metabolisms (Pizzarello and Shock 2010, and references there in).

Many experiments have been conducted with energy sources in the extraterrestrial environments to synthesize hydrocarbons, amino acids, and nucleic acid bases from simple molecules. A possible energy source for the formation of complex organic molecules could be an ultraviolet irradiation of dust grains in the interstellar medium. Muñoz Caro et al. (2002) performed simulation of interstellar ices mixtures of water, carbon dioxide, methanol, and ammonia, placed under a pressure of $10^{-7} \mathrm{mbar}$ at $12 \mathrm{~K}$ and irradiated with electromagnetic radiation, as well as their analysis by gas chromatography/mass spectrometry. They identified 16 amino acids, in the simulated ice mantle of interstellar dust particles, including 6 protein amino acids. Moreover, vacuum UV photoirradiation of simpler ices containing water, methanol, and ammonia led to the formation of hydantoin, 2,4imidazolidinedione (De Marcellus et al. 2011). The dominant amino acids were formed when 5-substituted hydantoins were UV-irradiated and hydrolyzed were Gly and Ala (Sarker et al. 2013). Kobayashi and coworkers simulated interstellar ice by using a starting material composed of a mixture of methanol, ammonia, and water (molar ratio $=1: 1: 2.8)$ and irradiated by heavy ions, and they hydrolyzed amino acids by using an ion-exchange high-performance liquid chromatography (IE-HPLC). A wide range of amino acids were detected after acid hydrolysis, including protein amino acids such as Ala, Gly, and Asp as well as non-protein amino acids such as $\beta$ alanine ( $\beta$-Ala) and $\alpha$ - and $\gamma$-aminobutyric acid (Kobayashi et al. 2012). Another possible scenario of the formation of organic matter is via aqueous alteration or a hydrothermal reaction in the meteorite parent bodies. A possible reaction for the formation of $\alpha$-amino acids is generally considered to be a Strecker-like reaction from precursor aldehydes, ketones, ammonia, and HCN in the presence of water. On the other hand, macromolecular organic solids can be produced from hydrothermal reaction of formaldehyde and yielded organic solids (Cody et al. 2011), and $\alpha-, \beta-$, and $\gamma$-amino acid precursors were produced simultaneously with organic solids when ammonia was added in the hydrothermal reaction of formaldehyde (Kebukawa et al. 2017).

\section{Stability of amino acids in hydrothermal environments}

Although, amino acids could be synthesized during aqueous alteration in meteorite parent bodies, continuous heating may have resulted in the decomposition of amino acids. It is known that less altered chondrites contains larger amounts of amino acids compared to highly altered or thermally metamorphosed chondrites, in which amino acids decay into monocarboxylic acids through the loss of their amine functional group (Elsila et al. 2016). So far, stability of amino acids in hydrothermal conditions have been studied in simulated submarine hydrothermal vents (e.g., Andersson and Holm 2000; Bada et al. 1995; Chandru et al. 2013; Cox and Seward 2007; Kohara et al. 1997; Kurihara et al. 2012). Kurihara et al. (2012) tested the stability of complex combined amino acid precursors, which were synthesized from a simulated primitive Earth atmosphere with proton irradiation, and showed that the complex combined amino acid precursors preserved more amino acids than free amino acids after heating in 
simulated submarine hydrothermal environments. However, stability and alteration processes of extraterrestrial amino acids and/or their precursors under hydrothermal conditions occurring in meteorite parent bodies have not yet been investigated well. The large amount of amino acids in primitive carbonaceous chondrites exists in the form of amino acid precursors (Glavin et al. 2010). In this study, we tested the stability and decomposition of the interstellar complex amino acid precursor analog during hydrothermal alteration simulating meteorite parent bodies under elevated temperatures.

\section{Methods}

\section{Materials and Samples}

Complex organic compounds were synthesized from a gas mixture of $\mathrm{CO}, \mathrm{NH}_{3}$, and $\mathrm{H}_{2} \mathrm{O}$, which are possible constituents of the interstellar medium, by irradiation with $2.5 \mathrm{MeV}$ protons from a Tandem accelerator (Tokyo Institute of Technology) following the method of Takano et al. (2004). Total energy applied to the gas mixture was $3.16 \mathrm{~kJ}$. The solution of synthesized complex organic compounds is slightly yellow and contains several thousand Daltons of amino acid precursors, which yielded various amino acids (protein and nonprotein amino acids) after acid hydrolysis (Takano et al. 2004). Hereafter, we simply called our amino acid precursor-containing solution synthesized from a gas mixture of $\mathrm{CO}, \mathrm{NH}_{3}$, and $\mathrm{H}_{2} \mathrm{O}$; the "AAP". We used the AAP as an analog of the primordial organic matter in primitive meteorite parent bodies to evaluate the stability of amino acids and their precursors.

\section{Autoclave Experiments}

To simulate hydrothermal conditions in meteorite parent bodies, an autoclave (AC), which is a closed hydrothermal system, was used in our hydrothermal experiments (Yanagawa and Kobayashi 1992; Kobayashi et al. 1995; Islam et al. 2002). It uses an electrothermal heater, in which the heating starts from room temperature (RT) to the desired temperature of $200-300{ }^{\circ} \mathrm{C}$. The inside temperature was monitored using thermocouples, and the pressure was maintained constant during the heating experiments.

The experimental procedures are summarized in Fig. 1a. The AAP was placed in a reaction tube, then settled the tube in the $\mathrm{AC}$, and the $\mathrm{AC}$ lid was closed firmly. To compare the effects of silicate and metal ion, both Pyrex glass tubes and stainless steel tubes were used as the reaction tubes in this study. The $\mathrm{AC}$ was purged with $\mathrm{N}_{2}$ or a gas mixture of $\mathrm{N}_{2}: \mathrm{H}_{2}(99: 1 \mathrm{vol} \%)$ to evaluate the effects of redox condition - the pressure valve was opened and

134 closed three times to eliminate all the gas inside the AC and was then pressurized to $8 \mathrm{MPa}$ at RT. The $\mathrm{N}_{2}+\mathrm{H}_{2}$ gas mixture provides more reduced condition than pure $\mathrm{N}_{2}$ gas. The pressure was increased to $14 \mathrm{MPa}$ with continuous

136 heating at high temperatures of 200,250 , and $300^{\circ} \mathrm{C}$. It took up to 30 minutes to reach to the target temperatures.

137 After the set temperature was reached, the heating of the $\mathrm{AC}$ was continued for $2 \mathrm{~h}$, after which the $\mathrm{AC}$ was left 138 to cool for approximately 30 minutes. A control AAP was also prepared by placing the solution in the AC under the same experimental conditions but without heating or pressurizing. 
The procedures for amino acid analysis are summarized in Fig. 1b. For amino acid analysis, after each autoclave run, an aliquot $(0.5 \mathrm{~mL})$ of the filtrated $\mathrm{AAP}$ was mixed with $0.5 \mathrm{~mL}$ of $12 \mathrm{M} \mathrm{HCl}$, and heated at $110^{\circ} \mathrm{C}$ for $24 \mathrm{~h}$

143 for acid hydrolysis. The acid hydrolysis method we applied here is selected due to: (i) Proteins and meteoritic 144 amino acids (precursors) have been acid hydrolyzed to evaluate amino acid composition by acid hydrolysis in 6 $145 \mathrm{M} \mathrm{HCl}$ at $100-110^{\circ} \mathrm{C}$ for 24 hours (Cronin and Pizzarello 1997; Simkus et al. 2019), and (ii) Our AAP has been 146 evaluated after the same acid hydrolysis conditions, and obtained fair results to evaluate the synthetic/alteration 147 reaction conditions. Under the previous classical hydrolysis conditions, most of amino acids are stable and they 148 can be determined quantitively (Davidson 2003; Rutherfurd and Gilani 2009). After acid hydrolysis, the AAP was 149 dried by centrifugation at $60^{\circ} \mathrm{C}$. The hydrolyzed fraction undergoes to desalting with cation-exchange resin (AG$15050 \mathrm{~W}-\mathrm{X} 8 \mathrm{resin})$ after dissolving in $2 \mathrm{~mL}$ of $0.1 \mathrm{M} \mathrm{HCl}$. The resin $(2-3 \mathrm{~mL})$ was prewashed using the following 151 liquids sequentially: $1 \mathrm{M} \mathrm{HCl}(20 \mathrm{~mL})$, water $(20 \mathrm{~mL}), 1 \mathrm{M} \mathrm{NaOH}(20 \mathrm{~mL})$, water $(20 \mathrm{~mL}), 1 \mathrm{M} \mathrm{HCl}(20 \mathrm{~mL})$, 152 and water $(20 \mathrm{~mL})$. An aliquot of $0.1 \mathrm{M} \mathrm{HCl}$ sample solution $(1 \mathrm{~mL})$ was introduced to the column after filtration 153 with polytetrafluoroethylene (PTFE) membrane filters (DISMIC-13HP $0.45 \mu \mathrm{m}$ ) and rinsed with $0.1 \mathrm{M} \mathrm{HCl}(1$ $154 \mathrm{~mL})$ and water $(20 \mathrm{~mL})$. Then, the solution containing amino acids was eluted with $10 \% \mathrm{NH}_{3}$ aqueous solution $155(15 \mathrm{~mL})$ and water $(20 \mathrm{~mL})$. The $\mathrm{NH}_{3}$ elute was dried by centrifugation at $60^{\circ} \mathrm{C}$, and the dried sample was dissolved in $0.5 \mathrm{~mL}$ of Milli-Q water. All the glassware used was baked at $500^{\circ} \mathrm{C}$ for at least $3 \mathrm{~h}$ before use in all experiments.

Before injection to the HPLC system, all the AAP samples were filtrated through cellulose acetate membrane filters (DISMIC-3CP $0.45 \mu \mathrm{m}$ ). A $100-\mu \mathrm{L}$ aliquot of each AAP solution was subjected to amino acid analysis by using an IE-HPLC system. The HPLC system was equipped with a system controller (Shimadzu SCL-10A), three HPLC pumps (two Shimadzu LC-20AD for carriers, and one LC-10ATvp for derivatization reagent) and a polystyrene-type ion-exchange column (Shimadzu Shim-pack ISC-07/S1504Na; $4.0 \mathrm{~mm}$ of inner diameter $\times 150$ $\mathrm{mm}$; particle diameter of $7 \mu \mathrm{m}$ ). The column temperature was conserved at $55^{\circ} \mathrm{C}$ by a column heater (Sugai U620 type 50). A fluorescence detector (Shimadzu RF-535) with an excitation wavelength of $355 \mathrm{~nm}$ and an emission wavelength of $435 \mathrm{~nm}$ was used. A post-column derivatization was used with a solution of ophthalaldehyde (OPA, $0.104 \mathrm{~g} / \mathrm{L}$ ) and N-acetyl-L-cysteine (NAC, $0.65 \mathrm{~g} / \mathrm{L}$ ), $\mathrm{Na}_{2} \mathrm{CO}_{3}(40.7 \mathrm{~g} / \mathrm{L}), \mathrm{H}_{3} \mathrm{BO}_{3}(13.5 \mathrm{~g} / \mathrm{L}$ ),

$166 \mathrm{~K}_{2} \mathrm{SO}_{4}(18.8 \mathrm{~g} / \mathrm{L})$, and polyoxyethylene lauryl ether $(0.2 \mathrm{~g} / \mathrm{L})$. Gradient elution was performed using a Shimadzu amino acid mobile phase kit (Na type): (A) $0.07 \mathrm{M}$ sodium citrate, hydrochloric peroxide, with $7 \%$ ethanol (pH 3.2); (B) $0.2 \mathrm{M}$ sodium citrate, boric acid, and sodium hydroxide ( $\mathrm{pH} \mathrm{10)}$; and (C) $0.2 \mathrm{M}$ sodium hydroxide. The

169 flow rate of the carrier was $0.3 \mathrm{~mL} / \mathrm{min}$. For peak identification, commercial amino acid standard solutions (Wako

170 Amino Acids Mixture Standard Solution, Type B, and Type AN-2) were used.

\section{$171 \quad$ UV absorption and UV fluorescence spectra}

Ultraviolet-visible (UV-Vis) spectroscopy was performed to investigate the electronic energy levels changes within the molecule arising due to the transfer of electrons from $\pi$ - or non-bonding orbitals. It usually provides

174 information about $\pi$-electron systems, conjugated unsaturation, aromatic compounds, and conjugated non-bonding 175 electron systems. This absorption spectroscopy uses electromagnetic radiations of wavelength $190-800 \mathrm{~nm}$, which is divided into the UV (190-400 nm) and Vis (400-800 nm) regions. 
An aliquot $(0.7 \mathrm{~mL})$ of each filtrated AAP was analyzed using a UV-VIS spectrometer (JASCO V-660). The absorption spectra were obtained with a wavelength range of 187-900 nm using a deuterium lamp (187-350 nm) and a halogen lamp (350-900 nm), with a wavelength accuracy of $\pm 1.5 \mathrm{~nm}$ and a spectral bandwidth of $2 \mathrm{~nm}$. The optical system was a double-beam with a double monochromator to provide higher resolution with extremely low stray light $(0.00008 \%)$ to confirm more accurate measurement over a wide photometric range. The UV absorption properties of the AAP were shown for a wavelength range of 200-400 nm.

The fluorescence intensities of $1 \mathrm{~mL}$ of the filtrated AAP were analyzed using a spectrofluorometer (JASCO FP-6300), equipped with a 150-watt Xe light source, a silicon photodiode detector for excitation monochromator, and a photomultiplier for the emission monochromator. The wavelength range was $220-700 \mathrm{~nm}$ with a standard photomultiplier tube, a wavelength accuracy of $\pm 2 \mathrm{~nm}$, and a spectral bandwidth of $20 \mathrm{~nm}$ for both excitation and emission monochromators. The UV fluorescence intensities of the AAP were determined at an excitation range of $220-600 \mathrm{~nm}$ and an emission range of $230-700 \mathrm{~nm}$.

\section{Results}

\section{Amino acid concentrations}

The concentrations of amino acids after acid hydrolysis of the heated AAP were quantified using HPLC. Figures 2 and 3 show chromatograms of the control AAP treated at RT, and the heated AAP at 200, 250, and $300{ }^{\circ} \mathrm{C}$ under $\mathrm{N}_{2}$ and $\mathrm{N}_{2}+\mathrm{H}_{2}$ in Pyrex glass tubes. A wide range of amino acids were detected after acid hydrolysis (Table 1). The control AAP exhibited the following amino acids: Gly and Ala with minor amount of Asp and serine (Ser) as well as non-protein amino acids; $\beta$-Ala, $\alpha$ - and $\gamma$-aminobutyric acid. For all the heated AAP at different temperatures $\left(200,250\right.$, and $\left.300^{\circ} \mathrm{C}\right)$, Gly and Ala were predominant, followed by $\alpha$ - and $\gamma$-aminobutyric acid, Ser, Asp, and $\beta$-Ala. After heating, amino acid concentrations decreased in general, but some amino acids increased with temperature. The recovery ratios (\%) of amino acids were obtained by the amino acid abundances from the heated AAP divided by those from the control AAP (treated at RT under the same experimental conditions) (Fig. 4). For the heated AAP under a gas mixture of $\mathrm{N}_{2}+\mathrm{H}_{2}$, Gly and Ala showed relatively high recovery ratios $(40 \%)$ at $200{ }^{\circ} \mathrm{C}$; this recovery ratio continued to decrease with increasing temperature and became only trace amounts at $300^{\circ} \mathrm{C}$. Asp and $\gamma$-aminobutyric acid showed different responses with heating compared to Gly and Ala. Asp showed a recovery ratio of $40 \%$ at $200{ }^{\circ} \mathrm{C}$, which continued to increase and became $60 \%$ at $300{ }^{\circ} \mathrm{C}$. $\gamma$-aminobutyric acid showed a high recovery ratio of $189 \%$ at $200{ }^{\circ} \mathrm{C}$, which increased efficiently to become $422 \%$ at $250{ }^{\circ} \mathrm{C}$, but finally, decreased to $144 \%$ at $300{ }^{\circ} \mathrm{C}$.

For the heated AAP under $\mathrm{N}_{2}$ gas, amino acids showed relatively lower recovery ratios than the heated AAP under a gas mixture of $\mathrm{N}_{2}+\mathrm{H}_{2}$, although they behave almost in the same manner in response to heating. For instance, the recovery ratios of Gly and Ala at $200{ }^{\circ} \mathrm{C}$ were $34 \%$ and $23 \%$, respectively, whereas their recovery ratios were greatly decreased and became $0.38 \%$ and $0.25 \%$, respectively, at $300{ }^{\circ} \mathrm{C}$. Moreover, the recovery ratio of Asp constantly increased from $33 \%$ at $200{ }^{\circ} \mathrm{C}$ to $43 \%$ at $300{ }^{\circ} \mathrm{C}$. Finally, $\gamma$-aminobutyric acid displayed again the same

211 behavior of an increased recovery ratio from $80 \%$ at $200{ }^{\circ} \mathrm{C}$ to $87 \%$ at $250{ }^{\circ} \mathrm{C}$, then a decreased ratio of $44 \%$ at $212300^{\circ} \mathrm{C}$. 
Gly and Ala decreased constantly with increasing temperature, but some amino acids showed a different

214 behavior, such as Asp and $\gamma$-aminobutyric acid, which increased with increasing temperature in some cases. At all

215 temperatures, the recovery ratio of each amino acid increased when hydrogen was added to the pressurizing gas.

Table 1. Amino acid concentrations and recovery ratios in the control AAP and the heated AAP after acid 218 hydrolysis.

\begin{tabular}{lcccccccc}
\hline & \multicolumn{7}{c}{$\mathrm{N}_{2}$, Pyrex } & \multicolumn{7}{c}{$\mathrm{N}_{2}+\mathrm{H}_{2}$, Pyrex } \\
Concentration $(\mu \mathrm{M})$ & Control & $200^{\circ} \mathrm{C}$ & $250^{\circ} \mathrm{C}$ & $300^{\circ} \mathrm{C}$ & Control & $200^{\circ} \mathrm{C}$ & $250^{\circ} \mathrm{C}$ & $300^{\circ} \mathrm{C}$ \\
\hline Glycine & 494.3 & 168.1 & 39.3 & 1.9 & 525.2 & 210.3 & 129.0 & 2.1 \\
Alanine & 126.1 & 28.9 & 0.4 & 0.3 & 154.4 & 61.8 & 5.9 & 2.0 \\
$\beta$-alanine & 2.7 & 0.4 & 0.2 & 0.1 & 3.0 & 1.1 & 0.6 & 0.3 \\
Aspartic acid & 3.9 & 1.3 & 1.2 & 1.7 & 4.3 & 1.7 & 2.0 & 2.6 \\
Serine & 5.7 & 1.0 & 0.2 & 0.1 & 7.7 & 2.3 & 0.8 & 0.8 \\
$\alpha$-aminobutyric acid & 7.8 & 4.0 & 2.8 & 0.9 & 10.9 & 12.0 & 8.2 & 2.2 \\
$\gamma$-aminobutyric acid & 0.7 & 0.5 & 0.6 & 0.3 & 2.0 & 3.8 & 8.4 & 2.9 \\
\hline Recovery ratio $(\%)$ & & $200^{\circ} \mathrm{C}$ & $250^{\circ} \mathrm{C}$ & $300^{\circ} \mathrm{C}$ & & $200^{\circ} \mathrm{C}$ & $250^{\circ} \mathrm{C}$ & $300^{\circ} \mathrm{C}$ \\
\hline Glycine & & 34 & 8.0 & 0.38 & & 40 & 25 & 0.40 \\
Alanine & & 23 & 0.34 & 0.25 & & 40 & 3.8 & 1.3 \\
$\beta$-alanine & 14 & 7.5 & 5.4 & & 36 & 19 & 10 \\
Aspartic acid & & 33 & 30 & 43 & & 40 & 46 & 60 \\
Serine & & 17 & 3.9 & 1.9 & & 30 & 10 & 10 \\
$\alpha$-aminobutyric acid & & 51 & 36 & 12 & & 110 & 75 & 20 \\
$\gamma$-aminobutyric acid & & 80 & 87 & 44 & & 189 & 422 & 144 \\
\hline
\end{tabular}

\section{UV absorption and UV fluorescence spectra}

221 Figure 5 shows the UV absorption spectra of the untreated AAP and the AAP after heating. The UV absorption

222 from 200 to $400 \mathrm{~nm}$ was mainly based on the presence of the aromatic carbon structure, while at 226-250 nm were

223 mainly caused by unsaturated $\pi-\pi^{*}$ transitions of the aromatic $\mathrm{C}=\mathrm{C}$ bond, while wide absorption peaks around

$224250-400 \mathrm{~nm}$ were due to the benzene ring structures with multiple conjugated structures $\mathrm{n}-\pi *$ and $\pi-\pi *$ transitions.

225 Both transitions require the presence of an unsaturated functional group (chromophore), such as $>\mathrm{C}=\mathrm{C},>\mathrm{C}=\mathrm{O}$, -

$226 \mathrm{~N}=\mathrm{O},>\mathrm{C}=\mathrm{N}-,-\mathrm{N}=\mathrm{N}-,>\mathrm{C}=\mathrm{N}-$, to provide the $\pi$ orbitals.

227 Subsequently, a broad peak is observed in the $250-300 \mathrm{~nm}$ region composed of multiple overlapping bands

228 from the aromatic structures. An absorption peak at 300-400 nm corresponds to $\mathrm{n}-\pi^{*}$ transitions of the $\mathrm{C}=\mathrm{O}$ bond.

229 The absorbance at the $220-350 \mathrm{~nm}$ region decreased as the temperature increased, and this behavior was almost

230 the same for heating under $\mathrm{N}_{2}$ and $\mathrm{N}_{2}+\mathrm{H}_{2}$ in Pyrex glass tubes. In the case of heating in stainless steel tubes,

231 although UV absorption of the AAP decreased with increasing temperature, each absorbance was higher than the

232 case of heating in the glass tube. 
In fluorescence analyses, a sample absorbs a photon to move to an excited state and emits a photon to return to the ground state. The intensity of the fluorescence is generally proportional to the concentration of organic 235 molecules. Figure 6 shows a three-dimensional UV fluorescence spectrum of the untreated AAP. All spectra are 236 plotted on the same emission/excitation wavelengths and intensity scales to allow comparison. 237 Typically, aromatic molecules exhibit strong and characteristic fluorescence when excited with UV. Figures 7-9 238 show three-dimensional UV fluorescence spectra of the heated AAP. Figure 10 shows the peak intensities of the 239 UV fluorescence spectra of the AAP, that show a slight increase with increasing the temperature.

\section{Discussion}

\section{Stabilities of amino acids}

In this experiment, we used the AAP obtained from simulating interstellar molecules and hydrothermal conditions simulating in meteorite parent bodies. In our AAP, the recovery ratios of Gly and Ala heated for $2 \mathrm{~h}$ at $200{ }^{\circ} \mathrm{C}$ were both $40 \%$ and became $25 \%$ and $3.8 \%$, respectively, after heating at $250{ }^{\circ} \mathrm{C}$; further, they reduced considerably to become $0.40 \%$ and $1.3 \%$, respectively, after heating at $300{ }^{\circ} \mathrm{C}$. These results are consistent with the experimental results of Kurihara et al. (2012), which heated a complex organic compound synthesized from a simulating early Earth atmosphere in the simulating submarine hydrothermal system - their results showed that the recovery ratios of Gly and Ala heated for $2 \mathrm{~h}$ at $200{ }^{\circ} \mathrm{C}$ were $60 \%$ and $68 \%$, respectively, whereas at $300{ }^{\circ} \mathrm{C}$, they became $0.4 \%$ and $3.4 \%$, respectively. Our results and Kurihara et al. (2012) both showed that amino acid concentrations decreased by heating due to the decomposition of amino acid precursor materials, and their stabilities decreased rapidly above $200{ }^{\circ} \mathrm{C}$. In the other words, complex organic compounds maintained their combined structure as AAP at $200{ }^{\circ} \mathrm{C}$ at least a part.

The stability of amino acids also depends on their functional groups. In this study, the concentrations of Gly, $\beta$-Ala, Asp, $\alpha$ - and $\gamma$-aminobutyric acid were relatively high after heating, while Ala and Ser were relatively low, indicating the differences in thermal lability among the amino acids in the AAP. Higher concentrations of Asp were observed in previous studies; for example, after heating pure seawater in a flow reactor for 20 minutes at $300{ }^{\circ} \mathrm{C}$ (Horiuchi et al. 2004) and natural samples collected from black and white smokers at temperatures $>200^{\circ} \mathrm{C}$ in the active hydrothermal systems (Pietrucci et al. 2018). Our results and the previous studies suggested that apparently thermal-refractory amino acids, such as Asp and $\gamma$-aminobutyric acid, remained in the form of complex macromolecular precursors even after heating at $200{ }^{\circ} \mathrm{C}$. Also, many previous studies suggested that combining amino acids with inorganic materials in hydrothermal solutions stabilizes them (Kawahata and Ishizuka 1993; Ito et al. 2006; Chandru et al. 2013). The stability of amino acids is also controlled by their ionic form (Pietrucci et al.

263 2018), which is dependent on $\mathrm{pH}$. Metal ions can also play a significant role in stabilizing labile amino acids via 264 complexation (Chandru et al. 2013). Differences of the results in the metal tube from those in the glass tube (Figs. 5 and 10) might reflect the possible roles of metal ion.

The recovery ratios of $\gamma$-aminobutyric acid was considerably greater than any amino acids, and the recovery ratios reach up to $>400 \%$. It suggests not only high thermal stability of $\gamma$-aminobutyric acid but also its formation. 
condensates of $\gamma$-aminobutyric acid upon being heated (Scheme 1, Islam et al. 2003) and the rings were opened after acid hydrolysis. The $\omega$-amino acids could be chemical markers of abiotic hydrothermal synthesis.

271 Hydrothermal alteration in the amino acid composition by heating has been observed, where $\gamma$-aminobutyric acid 272 increased with temperature increasing from $200^{\circ} \mathrm{C}$ to $250^{\circ} \mathrm{C}$, and then decreased again at $300{ }^{\circ} \mathrm{C}$.

273

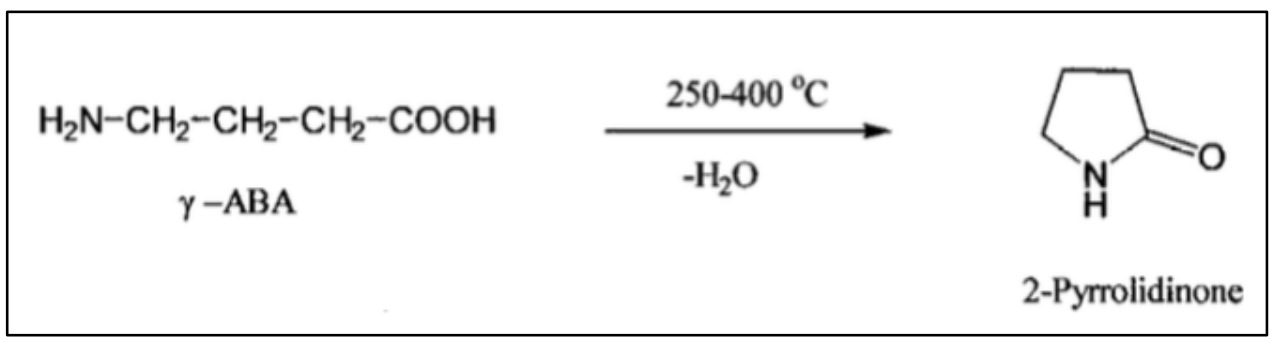

(Scheme 1)

The amino acid recovery ratios from the AAP are different from the reported values of recovery ratios of free amino acids. Kohara et al. (1997) heated an aqueous solution of amino acids at $200-350{ }^{\circ} \mathrm{C}$ for $2 \mathrm{~h}$ using an AC and showed that the recovery ratios of Gly and Ala heated at $200{ }^{\circ} \mathrm{C}$ were $\sim 105 \%$ and $\sim 95 \%$, respectively, which then decreased after heating at $250{ }^{\circ} \mathrm{C}$ to $\sim 85 \%$ and $\sim 100 \%$, respectively, and at $300{ }^{\circ} \mathrm{C}$, the recovery ratios became $\sim 80 \%$ and $\sim 50 \%$, respectively. Kobayashi at al. (1995) heated an aqueous solution of amino acids at $300{ }^{\circ} \mathrm{C}$ for 2 $\mathrm{h}$ using an $\mathrm{AC}$ and showed that the recovery ratios of Gly and Ala were $72 \%$ and $51 \%$, respectively. The recovery ratios of Gly and Ala in their studies were much higher than those in our studies; this may be due to the higher $\mathrm{pH}$ in our study (pH 3.6 for Kohara et al. vs. neutral $\mathrm{pH}$ for our study) and/or the higher concentration of Gly and Ala in their starting material, which was $50 \mu \mathrm{M}$ each, whereas the concentrations of Gly and Ala in the control AAP (at RT) was $30 \mu \mathrm{M}$ and $12.8 \mu \mathrm{M}$, respectively. In our study, the recovery ratios of Gly and Ala at $200{ }^{\circ} \mathrm{C}$ were $40 \%$, and the values gradually decreased to $25 \%$ and $3.8 \%$, respectively, at $250{ }^{\circ} \mathrm{C}$. At $300{ }^{\circ} \mathrm{C}$, the recovery ratios of Gly and Ala showed only trace amounts, $0.4 \%$, and $1.3 \%$, respectively. The recovery ratios of Asp and Ser in Kohara et al.'s studies were lower than those of amino acids of this study. In this study, the recovery ratios of Asp and Ser after the AAP heated at $300^{\circ} \mathrm{C}$ were $60 \%$ and $10 \%$, respectively; however, in Kobayashi et al. (1995), the recovery ratios were $0.41 \%$ and under detection limit, respectively, and in Kohara et al. (1997), trace amounts of Asp and Ser were recovered after $300^{\circ} \mathrm{C}$.

The amino acid recovery ratios were affected by the purged gas compositions. The recovery ratio was higher when the used pressurized gas was a mixture of nitrogen (99\%) and hydrogen (1\%) than when a pure nitrogen was used. Thus, it was suggested that amino acid stabilities were higher in reduced conditions. This is consistent with the results of Kohara et al. (1997) in the simulated submarine hydrothermal vent, which showed that the amino acids' recovery ratio was increased when hydrogen was added to the environmental gas.

Lastly, the AAP provided various amino acids after acid hydrolysis with high concentrations of Gly, and Ala, where they were decomposed with increasing temperature. Moreover, some amino acids released from the AAP, such as $\gamma$-aminobutyric acid, which increased with increasing temperature; this indicates that some amino acids were produced by hydrothermal alteration. 
UV absorption spectra of the AAP that is treated in the AC indicated that it decomposed with increasing temperature. Results in Fig. 5 showed that the absorption of the AAP at 226-400 nm decreased constantly with increasing temperature, indicating that conjugated bonds such as olefinic and aromatic compounds decreased. Typically, organic compounds degraded more easily, and its stability was relatively weaker if there were fewer aromatic structures in the organic molecules. Therefore, the AAP lost its stability gradually during heating, and the molecular weight of organic matters decreased accordingly.

Generally, the heated AAP in either the Pyrex glass tube (both in $\mathrm{N}_{2}$ and $\mathrm{N}_{2}+\mathrm{H}_{2}$ ) or the stainless steel tube, showed a decrease in the UV absorption at 230-400 nm with increasing temperature, indicating that organic compounds were decomposed with increasing the temperature. Decreases in the UV absorption of the AAP by heating in stainless steel tubes were less than those in Pyrex glass tubes. Metal ions from the stainless steel tube can act on the organic compounds and form complexes serving as electron donors or acceptors. Metal ions are necessary for the origin of life on the Earth as they enhance the stability of organic compounds through supporting reactions via catalytic effects and/or stabilizing organic molecules (Chandru et al. 2013; Pearson et al. 2002).

In UV fluorescence analysis, the peak intensities of the AAP generally increased with increasing the temperature. Some cyclic compounds such as aromatic and/or heterocyclic compounds were suggested to form with increasing temperature. When stainless steel tubes were used, the peak intensities were considerably lower than those when Pyrex glass tubes were used. In the case of using the Pyrex glass tube, silica could combine with organics, forming complex organic compounds, while in the case of using stainless steel tube, metal ions could combine with organics, forming complex organic compounds. Thus, the presence of silica may enhance the formation of aromatic and/or heterocyclic compounds.

\section{Implications for meteorite parent body alteration}

Primitive carbonaceous chondrites experienced aqueous alteration. Temperatures of aqueous alteration varied for the different meteorite groups and were estimated as $20-150{ }^{\circ} \mathrm{C}$ for $\mathrm{CI}, 0-80^{\circ} \mathrm{C}$ for $\mathrm{CM} 2,50-150{ }^{\circ} \mathrm{C}$ for $\mathrm{CR}$, and $50-340^{\circ} \mathrm{C}$ for $\mathrm{CV}$ (Brearley 2006 and references therein). Many attempts have been made to investigate the chemical evolution of insoluble organic matter (IOM) (Quirico et al. 2014; Cody and Alexander 2005; Alexander et al. 2007, 2010; Kebukawa et al. 2011; Orthous-Daunay et al. 2013; Sephton et al. 2000, 2004) and soluble organic matter (SOM) (Martin and Sephton 2010; Glavin et al. 2010; Burton et al. 2012b; Elsila et al. 2016) in various carbonaceous chondrites during hydrothermal alteration. In this study, we investigated hydrothermal alteration of soluble organic matter in environments simulating parent bodies of meteorites by using an AC, which is considered to be a closed hydrothermal system, especially to evaluate the effects of fluid temperatures on recovery of amino acids, although the effects of pressure, concentration, metal ions, and $\mathrm{pH}$ were not well considered in the current study. As the results, the recovery ratios of Gly and Ala heated at $200{ }^{\circ} \mathrm{C}$ were up to $\sim 40 \%$. Considering that the alteration temperatures of $\mathrm{CI}, \mathrm{CM}$, and $\mathrm{CR}$ chondrites are up to $150^{\circ} \mathrm{C}$ (Brearley 2006), our results are consistent with that the amino acids are abundant in non-heated carbonaceous chondrites $(\mathrm{CI} / \mathrm{CM} / \mathrm{CRs}$ ) but drastically decreased in heated chondrites (e.g., CVs, COs, and heated CMs) (Elsila et al. 2016). Heating the AAP at high temperature brought the increasing of $\gamma$-aminobutyric acid-this esult was dso consistent with the fact that relatively high abundances of $\omega$-amino acids, including $\gamma$-aminobutyric acid, in heated 
chondrites (Elsila et al. 2016). In CI, CM, and CR chondrites, 38-64\% of amino acids were detected without acid hydrolysis ("free" amino acids) and the rest ("bonded" amino acids) were only detectable after acid hydrolysis (Glavin et al. 2010), while only 13\%-33\% of total amino acids in thermally metamorphosed CV and CO chondrites were free (except one CV with 60\% free amino acids) (Burton et al. 2012a). Our study indicated that a highmolecular-weight complex organic compound AAP synthesized from simulated interstellar gas maintained their combined structure as amino acid precursors after heating at more than $200{ }^{\circ} \mathrm{C}$. It can partly explain the high abundances of "bonded" amino acids in meteorites, particularly in heated ones.

\section{Conclusions}

Hydrothermal experiments using an autoclave (AC) were conducted with simulated interstellar complex organic compounds synthesized from carbon monoxide, ammonia, and water as an analog of complex amino acid precursors ("AAP") to evaluate their stabilities. The AAP is known to produce various amino acids (predominantly Gly and Ala) after acid hydrolysis. Amino acids released after the heating experiments decreased with increasing temperature, but approximately $40 \%$ of Gly and Ala could survive at $200{ }^{\circ} \mathrm{C}$. However, $\gamma$-aminobutyric acid increased after heating under a gas mixture of $\mathrm{N}_{2}+\mathrm{H}_{2}$, indicating that $\gamma$-aminobutyric acid was synthesized during hydrothermal alteration. Finally, our data showed that the molecular structures of the AAP could be altered to form organic compounds that are more stable and can survive in elevated hydrothermal systems on meteorite parent bodies.

\section{Acknowledgements}

The present authors thank to Ms. Midori Eto (Yokohama National University) for her kind help in the proton irradiation experiments. We thank two anonymous reviewers for constructive comments which helped to improve the manuscript. The present work was partly supported by JSPS KAKEHNI Grant Number JP17H02991, JP17H06458 and JP18K03722.

\section{References}

Alexander CMO, Fogel M, Yabuta H, Cody GD (2007) The origin and evolution of chondrites recorded in the elemental and isotopic compositions of their macromolecular organic matter. Geochimica et Cosmochimica Acta 71:4380-4403

Alexander CMO, Newsome SD, Fogel ML, et al (2010) Deuterium enrichments in chondritic macromolecular material-Implications for the origin and evolution of organics, water and asteroids. Geochimica et Cosmochimica Acta 74:4417-4437

Anders E (1989) Pre-biotic organic matter from comets and asteroids. Nature 342:255

Bar-Nun A, Bar-Nun N, Bauer SH, Sagan C (1970) Shock Synthesis of Amino Acids in Simulated Primitive Environments. Science 168:470-472

Bar-nun A, Hartman H (1978) Synthesis of organic compounds from carbon monoxide and water by UV photolysis. Origins Life Evol Biosphere 9:93-101

Brearley AJ (2006) The Action of Water. In: Meteorites and the Early Solar System II. (eds. D. S. Lauretta, J. H. Y. McSween). University of Arizona Press, Tucson, pp 584-624

Burton AS, Elsila JE, Callahan MP, et al (2012a) A propensity for n- $\omega$-amino acids in thermally altered Antarctic meteorites: $n-\omega$-amino acids in thermally altered meteorites. Meteoritics \& Planetary Science 47:374-386

Burton AS, Stern JC, Elsila JE, et al (2012b) Understanding prebiotic chemistry through the analysis of extraterrestrial amino acids and nucleobases in meteorites. Chem Soc Rev 41:5459 
Chandru K, Imai E, Kaneko T, et al (2013) Survivability and Abiotic Reactions of Selected Amino Acids in Different Hydrothermal System Simulators. Origins Life Evol Biosphere43:99-108

Chyba C, Sagan C (1992) Endogenous production, exogenous delivery and impact-shock synthesis of organic molecules: an inventory for the origins of life. Nature 355:125

Cleaves HJ (2012) Prebiotic chemistry: What we know, what we don't. Evol Educ Outreach 5:342-360

Cody GD, Alexander CMO’D. (2005) NMR studies of chemical structural variation of insoluble organic matter from different carbonaceous chondrite groups. Geochimica et Cosmochimica Acta 69:1085-1097

Cody GD, Heying E, Alexander CMO, et al (2011) Establishing a molecular relationship between chondritic and cometary organic solids. PNAS 108:19171-19176

Cox JS, Seward TM (2007) The reaction kinetics of alanine and glycine under hydrothermal conditions. Geochimica et Cosmochimica Acta 71:2264-2284

Cronin JR, Pizzarello S (1997) Enantiomeric excesses in meteoritic amino acids. Science 275:951-955

Davidson I (2003) Hydrolysis of Samples for Amino Acid Analysis. In: Smith BJ (ed) Protein Sequencing Protocols. Humana Press, Totowa, NJ, pp 111-122

De Marcellus P, Bertrand M, Nuevo M, et al (2011) Prebiotic Significance of Extraterrestrial Ice Photochemistry: Detection of Hydantoin in Organic Residues. Astrobiology 11:847-854

Elsila JE, Aponte JC, Blackmond DG, et al (2016) Meteoritic Amino Acids: Diversity in Compositions Reflects Parent Body Histories. ACS Cent Sci 2:370-379

Glavin DP, Callahan MP, Dworkin JP, Elsila JE (2010) The effects of parent body processes on amino acids in carbonaceous chondrites. Meteoritics \& Planetary Science 45:1948-1972

Harada K, Fox SW (1964) Thermal Synthesis of Natural Amino-Acids from a Postulated Primitive Terrestrial Atmosphere. Nature 201:335-336

Horiuchi T, Takano Y, Ishibashi J, et al (2004) Amino acids in water samples from deep sea hydrothermal vents at Suiyo Seamount, Izu-Bonin Arc, Pacific Ocean. Organic Geochemistry 35:1121-1128

Islam MN, Kaneko T, Kobayashi K (2002) Determination of Amino Acids Formed in a Supercritical Water Flow Reactor Simulating Submarine Hydrothermal Systems. Analytical Sciences/Supplements 17icas: i1631i1634

Islam MN, Kaneko T, Kobayashi K (2003) Reaction of Amino Acids in a Supercritical Water-Flow Reactor Simulating Submarine Hydrothermal Systems. Bulletin of the Chemical Society of Japan 76:1171-1178

Ito M, Gupta LP, Masuda H, Kawahata H (2006) Thermal stability of amino acids in seafloor sediment in aqueous solution at high temperature. Organic Geochemistry 37:177-188

Kasting JF, Brown LL (1998) The early atmosphere as a source of biogenic compounds. In: A. Brack (ed.) The Molecular Origins of Life: Assembling Pieces of the Puzzle. Cambridge Univ Press, New York, pp.35-56

Kawahata H, Ishizuka T (1993) Amino-acids in the sediments and interstitial waters from ODP hole-677b and hole-678b in the panama basin. Oceanologica. Acta 16:373-379

Kebukawa Y, Alexander CMO, Cody GD (2011) Compositional diversity in insoluble organic matter in type 1, 2 and 3 chondrites as detected by infrared spectroscopy. Geochimica et Cosmochimica Acta 75:3530-3541

Kebukawa Y, Chan QHS, Tachibana S, et al (2017) One-pot synthesis of amino acid precursors with insoluble organic matter in planetesimals with aqueous activity. Sci Adv 3: e1602093

Kobayashi K, Kaneko T, Saito T (1999) Characterization of complex organic compounds formed in simulated planetary atmospheres by the action of high energy particles. Advances in Space Research 24:461-464

Kobayashi K, Kaneko T, Saito T, Oshima T (1998) Amino Acid Formation in Gas Mixtures by High Energy Particle Irradiation. Origins Life Evol Biosphere 28:155-165

Kobayashi K, Kasamatsu T, Kaneko T, et al (1995) Formation of amino acid precursors in cometary ice environments by cosmic radiation. Advances in Space Research 16:21-26

Kobayashi K, Kohara M, Gamo T, Yanagawa H (1995) Formation and alteration of organic compounds in simulated submarine hydrothermal vent environments. In: Sakai H, Nozaki Y (ed.) Bioorganic processes and the Ocean Flux in the Western Pacific. Terrapub, Tokyo, pp 532-535

Kobayashi K, K. Sarker P, Ono K, et al (2012) Formation, Alteration and Delivery of Exogenous High Molecular Weight Organic Compounds: Objectives of the Tanpopo Mission from the Point of View of Chemical Evolution. Aerospace Technology Japan 10: Tp_7-Tp_11

Kohara M, Gamo T, Yanagawa H, Kobayashi K (1997) Stability of Amino Acids in Simulated Hydrothermal Vent Environments. Chem Lett 26:1053-1054

Kurihara H, Takano Y, Kaneko T, et al (2012) Stability of Amino Acids and Related Compounds in Simulated Submarine Hydrothermal Systems. BCSJ 85:624-630

Martins Z, Sephton MA (2010) Extraterrestrial Amino Acids. In: Amino Acids, Peptides and Proteins in Organic Chemistry. John Wiley \& Sons, Ltd, pp 1-42 
McCollom TM, Lollar BS, Lacrampe-Couloume G, Seewald JS (2010) The influence of carbon source on abiotic organic synthesis and carbon isotope fractionation under hydrothermal conditions. Geochimica et Cosmochimica Acta 74:2717-2740

Miller SL (1953) A Production of Amino Acids Under Possible Primitive Earth Conditions. Science 117:528-529

Miller SL, Urey HC (1959) Organic compound synthesis on the primitive earth. Science 130:245-251

Muñoz Caro GM, Meierhenrich UJ, Schutte WA, et al (2002) Amino acids from ultraviolet irradiation of interstellar ice analogues. Nature 416:403-406

Oró J (1961) Comets and the formation of biochemical compounds on the primitive Earth. Nature 190:389-390.

Orthous-Daunay F-R, Quirico E, Beck P, et al (2013) Mid-infrared study of the molecular structure variability of insoluble organic matter from primitive chondrites. Icarus 223:534-543

Pearson VK, Sephton MA, Kearsley AT, et al (2002) Clay mineral-organic matter relationships in the early solar system. Meteoritics \& Planetary Science 37:1829-1833

Pietrucci F, Aponte JC, Starr R, et al (2018) Hydrothermal decomposition of amino acids and origins of prebiotic meteoritic organic compounds. ACS Earth Space Chem 2:588-598

Pizzarello S, Cooper GW, Flynn GJ (2006) The nature and distribution of the organic material in carbonaceous chondrites and interplanetary dust particles. In Meteorites and the Early Solar System II (eds. Lauretta DS, McSween JHY). University of Arizona Press, Tucson, pp. 625-651

Pizzarello S, Shock E (2010) The organic composition of carbonaceous meteorites: the evolutionary story ahead of biochemistry. Cold Spring Harbor Perspectives in Biology 2:a002105

Quirico E, Orthous-Daunay F-R, Beck P, et al (2014) Origin of insoluble organic matter in type 1 and 2 chondrites: New clues, new questions. Geochimica et Cosmochimica Acta 136:80-99

Rutherfurd SM, Gilani GS (2009) Amino acid analysis. Current Protocols in Protein Science 58: 11.9.1-11.9.37

Sagan C, Khare BN (1971) Long-Wavelength Ultraviolet Photoproduction of Amino Acids on the Primitive Earth. Science 173:417-420

Sarker PK, Takahashi J, Obayashi Y, et al (2013) Photo-alteration of hydantoins against UV light and its relevance to prebiotic chemistry. Advances in Space Research 51:2235-2240

Scattergood T (1989) Production of organic compounds in plasmas: A comparison among electric sparks, laserinduced plasmas, and UV light. Icarus 81:413-428

Sephton MA, Love GD, Watson JS, et al (2004) Hydropyrolysis of insoluble carbonaceous matter in the Murchison meteorite: new insights into its macromolecular structure11Associate editor: G. D. Cody. Geochimica et Cosmochimica Acta 68:1385-1393

Sephton MA, Pillinger CT, Gilmour I (2000) Aromatic moieties in meteoritic macromolecular materials: analyses by hydrous pyrolysis and $\delta 13 \mathrm{C}$ of individual compounds. Geochimica et Cosmochimica Acta 64:321-328

Simkus DN, Aponte JC, Elsila JE, et al (2019) Methodologies for analyzing soluble organic compounds in extraterrestrial samples: Amino acids, amines, monocarboxylic acids, aldehydes, and ketones. Life 9:47

Stribling R, Miller SL (1987) Energy yields for hydrogen cyanide and formaldehyde syntheses: The HCN and amino acid concentrations in the primitive ocean. Origins Life Evol Biosphere 17:261-273

Takano Y, Ohashi A, Kaneko T, Kobayashi K (2004) Abiotic synthesis of high-molecular-weight organics from an inorganic gas mixture of carbon monoxide, ammonia, and water by $3 \mathrm{MeV}$ proton irradiation. Appl Phys Lett 84:1410-1412

Walker JCG (1977) Evolution of the Atmosphere. Maxwell Macmillan Canada

Yanagawa H, Kobayashi K (1989) Formation of amino acids, peptide-like polymers, and microspheres in superheated hydrothermal environments. Origins Life Evol Biosphere 19:540-541

Yanagawa H, Kobayashi K (1992) An Experimental Approach to Chemical Evolution in Submarine Hydrothermal Systems. In: Holm NG (ed) Marine Hydrothermal Systems and the Origin of Life. Springer Netherlands, Dordrecht, pp 147-159 